\title{
Research and Design of a Fusion Technology in Highway Engineering Measurement and Payment System
}

\author{
Junqing Liang ${ }^{1, ~ a, ~ S h a o h u a ~ Z h a o ~}{ }^{2, b}$, Jinlong Wang ${ }^{1, \text { a }}$ and Zhongguo $\mathrm{Du}^{1, \mathrm{c}}$ \\ ${ }^{1}$ School of Computer Engineering, Qingdao Technological University, Qingdao, China \\ ${ }^{2}$ Qingdao Letong Construction Group, Qingdao, China \\ a junqingl@163.com, b zhaoshaohua6167@163.com, ' wangjinlong@gmail.com, \\ dduzhg_1126@163.com
}

Keywords: Highway Engineering; Measurement and Payment; Workflow; GIS; Fusion Technology

\begin{abstract}
For the request that was proposed in research and design for measurement research and payment system of the highway engineering: flexible business process control and integration management issues of spatial and attribute data and so on. The article proposed a method in which GIS integrated with workflow: using construction supervision for highway measurement and payment workflow as main line, using component software technology as basis, and with a development platform achieving the integration of workflow with GIS. Based on the fusion technology, the article completed the technical framework and design of the system. The feasibility and effectiveness of this fusion technology was verified in the practical application.
\end{abstract}

\section{Introduction}

With the rapid economic development and the gradual expansion of computer applications, The awareness and research of construction supervision for highway information was gradually developed and improved. At present, The supervision of highway construction measurement and payment system being applied utilized workflow thought going on defining and modeling for the existing mature and solid business process of Supervision of highway construction measurement and payment. It can realize information sharing, standardize process control and further meet the needs of different users or different projects. It can also improve management and ensure high quality and efficient project completion. The roads are linear structures with spatial characteristics and distinctive geographical features. Only using attribute data in text form to express the information is not enough. So in order to effectively grasp the highway resources and understand comprehensively road layout, road data should be linked with geographical information. In other words, the road traffic, environment along the road and the progress of highway construction and other information should be able to graphically display in the form of spatial data. Thus, it is visual image and clear. The geographic information system(referred to as GIS) technology implements a combination of graphics and data. It is able to provide a new management approach for three different users in the form of data visualization. In view, construction supervision in highway Engineering Measurement and Payment System not only requires to control workflow flexibly, but also requires to manage attribute data, spatial data and other multi-source data. To this end, taking advantage of the combination between workflow technology and GIS, combining with the development trend of software technology, a solution of a fusion method was proposed: using construction supervision for highway measurement and payment workflow as main line, using component software technology as basis , and with a development platform achieve the integration of workflow with GIS.

\section{The Solutions to Select the Fusion Technology}

There are two cases for Technology and technology integration. First, it is to the theory supporting the technology or theoretical system to expand, so that it can accommodate the technology to be integrated and the theory supporting the technology or theoretical system. Then it is to develop the new technologies under a new theory or theoretical system. This new technology combines the 
characteristics and functions of the old technology. Second, it is to build or through a platform or a tool that enables the technology to be integrated from a whole under the inclusion platform or tools. It provides variety of function and service which is supported by variety of technology. Obviously, the first is the field of workflow and experts or academics in the field of GIS to consider. Our aim is to consider using the existing workflow technology and GIS technology, and even the professional software tools to develop an application system with workflow and GIS functional characteristics in the actual project for development and construction of the system. This is the second case to cover. In study design for construction supervision in highway Engineering Measurement and Payment System, in order to fuse workflow and GIS technology and develop the application system with management function of workflow and GIS. There are three basic types of technology ideas.

A. "independent type" fusion idea. Using the existing software system or developed GIS functional program modules, connect to workflow application system through the way of file link and achieve the fusion of two types of technology in form.

B. "inclusive type of mother and child" fusion ideas. In supporting of OLE automation technology and COM technology workflow application development platform to introduce GIS application components, achieving the fusion of workflow and GIS technology by programming.

C."self-contained type" fusion ideas. With powerful program development capabilities of common application development platform, getting rid of all or most of the dependence on existing software systems, developing independently complex application system with functional characteristics of workflow automation and GIS.

\section{Research and Design of Fusion Technology in Highway Engineering Measurement and Payment System}

In the full study of the "independent type", "inclusive type of mother and child" and "self-contained type" three basic workflow and GIS fusion ideas, and considering the supervision of high construction measurement and payment system under actual conditions, we proposed the "using construction supervision for highway measurement and payment workflow as main line, using component software technology as basis , with Microsoft Visual.net platform" to achieve the fusion of workflow and GIS and the line for system development.

\section{A. The Two Levels for The Integration of Workflow and GIS}

Based on the analysis of the first three fusion ideas and the comparison and research of the corresponding development tools, combined with the actual system requirements and the gradual improvement of the road environment and conditions for current construction supervision, we made a comprehensive analysis of the characteristics of many development tools, and then selected Microsoft Visual.net + MapObjects as the integration platform to study, design and develop the subject. Specifically, MapObjects used the industry standard COM architecture. So it had an industry standard "agreement" or "interface" with Microsoft Visual.net, models and so on It achieved easily the seamless connectivity and integration.

1) Integrated fusion of activity level

By general visual development tools Microsoft Visual.net of supporting component technology, we established the basic framework for application system and developed the workflow engines. We used graphic processing function implemented by GIS as an activity in workflow controlled by the workflow.

2) Integrated fusion of tools level

We used component GIS to develop graphics processing tools for business activity use. The business activities were still controlled by the unified Workflow Management.

The following figure shows the technology framework of the fusion for GIS and workflow. 


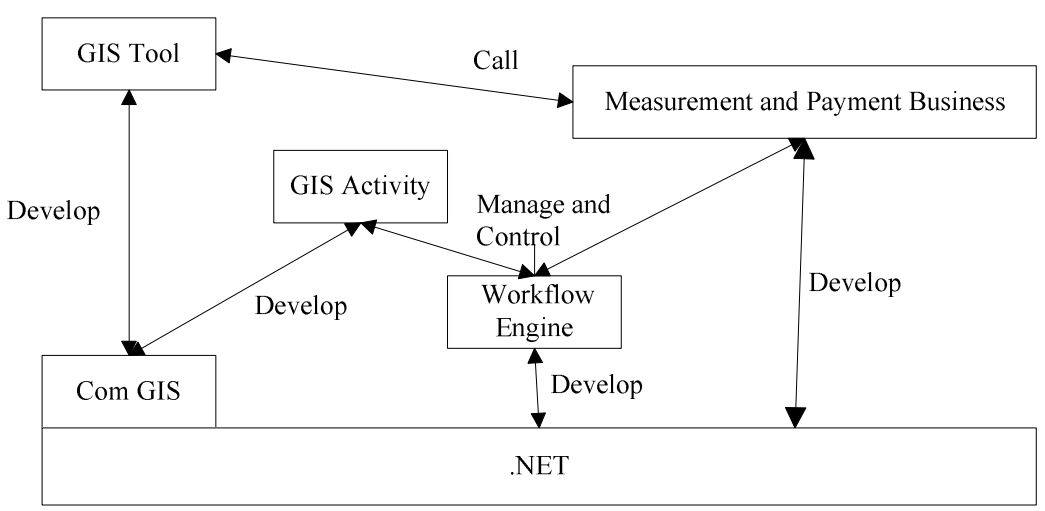

Fig. 1. The technology framework for fusion of workflow and GIS

\section{B. Workflow and Data Organization and Management in GIS Integration and Call Relationship}

According to system functions, the data involved can be divided into three parts: management data, business data and map data. Management data is the control data which is required by the workflow definition, management and implementation, including management data for users, roles, rights, management of custom data process management of status tracking data and management of view data, etc. Business data includes static management data of project data, dynamic production data in business programs and business inquiry or statistics data, etc. Map data are divided into two parts. One is graphic data. The second is the attribute data.

The below is the call graph of data:

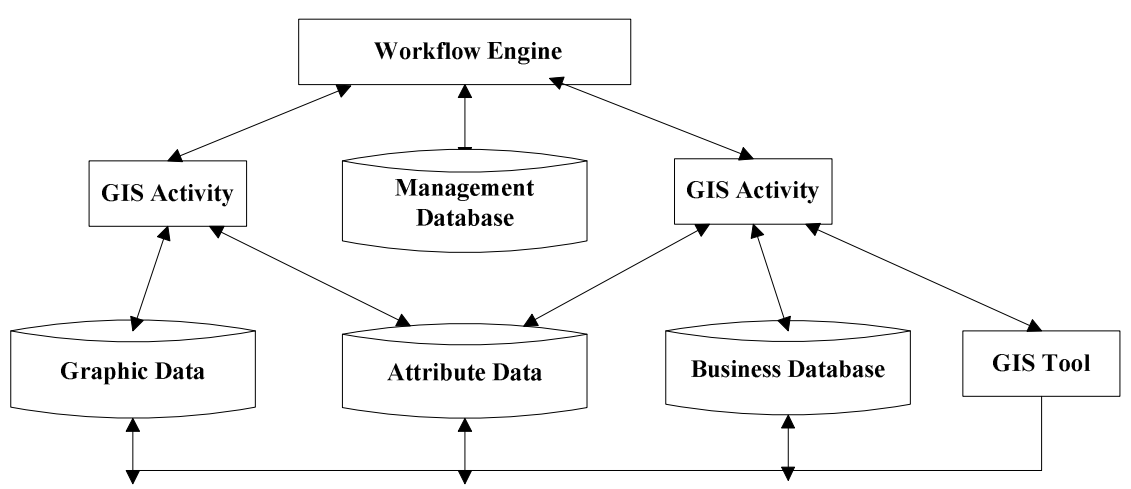

Fig. 2. The data call graph for the fusion of workflow and GIS

According to the integrated fusion method which was described and used in the previous subject, data management and call method, GIS function can be integrated into the workflow management. We conducted the architecture design following these two principles as follows.

1)Adopt distributed environment, three-tier distributed architechture

From the view that the workflow technology needs to solve problem, it must be appeared in the form of distribution. The subject follows this. Logically, according to the data service layer, business logic layer, user interface, the system is divided into the database server, application program server and client applications.

2)The separation of GIS service and non-GIS service

Throughout the system, GIS is embedded in it as a component. As an activity, it is called by the workflow engine. As the interface of access data, the application of data and display mechanisms for GIS service and non-GIS service are different, so the two kind of service are separated effectively on the application program server in the system. After separation, it is easy to implement and facilitate the client's call.

Thus, we referred to WFMC reference model architecture, giving the following system architecture. 


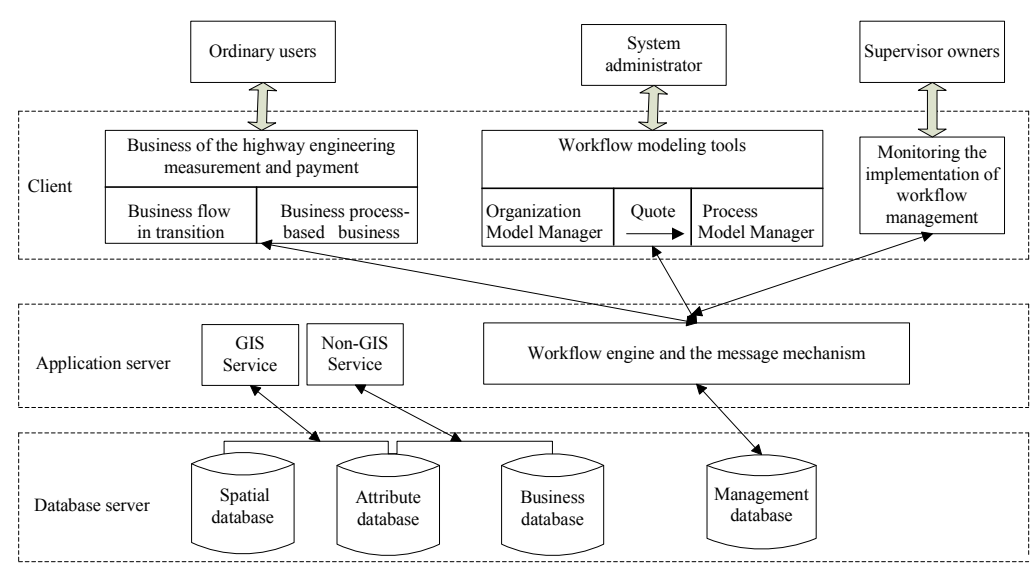

Fig. 3. The architecture for measurement and payment workflow of highway engineering and integrated fusion GIS

\section{Summary and Outlook}

The article analyzed the idea that workflow fused GIS technology, proposed a fusion technology for the system and applied to the study and design of the supervision of highway construction measurement and payment system. And to design method and technology implementation, it carried out to verify feasibility in the landscape road construction supervision measurement and payment of Zouping It achieved good results. In the future, we will focus on the deep meaning of the three fusion theory and application to do more in-depth research and analsis. So as to building highway construction supervision for the construction started, decision-making, construction and completion of the entire process, as well as provide an effective guarantee for scientific, standardized and automated management. The fusion technology application of GIS and workflow will play the role of producing better ideas for the entire industry's information system construction of road construction supervision.

\section{References}

[1] WfMC, The workflow reference model[S], 1995.

[2] S.X. Huang, F. Gao, Research on integration technology of workflow automatization with GIS in electronic government system[J], Journal of hefei university of technology(natural science), 2004, 27(2):13-15.

[3] Z.X Liao, F. Gao, Research on integration technology of workflow automatization with GIS in electronic government system[J], Journal of hefei university of technology(natural science), 2005, 28(3):35-37.

[4] W. Xue, MapObjects Geographic Information System Programming, Beijing: National Defence Industrial Press, 2004, 1:1-2.

[5] G. Liu, X.D. Liu, Secondary Development Tutorial Examples of Geographic Information System, Tsinghua University Press, 2004.

[6] X.X Liao, Research on implementation of electronic government affairs for workflow fused with GIS technology(Master thesis), Hefei: HeFei University of Technology, 2005.

[7] ESRI Company, ArcSDE Configuration and Tuning Guide for MS SQL Server[R], 2002:11. 